\title{
Erratum: The genome and transcriptome of the zoonotic hookworm Ancylostoma ceylanicum identify infection-specific gene families
}

Erich M Schwarz, Yan Hu, Igor Antoshechkin, Melanie M Miller, Paul W Sternberg \& Raffi V Aroian Nat. Genet. 47, 416-422 (2015); published online 2 March 2015; corrected after print 5 May 2015

In the version of this article initially published, the following two sentences were omitted from the Acknowledgments: "Sequencing was carried out at the Millard and Muriel Jacobs Genome Facility at the California Institute of Technology. This work was supported by US National Institutes of Health grants to P.W.S. (GM084389) and to R.V.A. (AI056189), by Cornell University salary and start-up funds to E.M.S. and by the Howard Hughes Medical Institute to P.W.S." The error has been corrected in the HTML and PDF versions of the article.

Corrigendum: Analysis of the genetic phylogeny of multifocal prostate cancer identifies multiple independent clonal expansions in neoplastic and morphologically normal prostate tissue

Colin S Cooper, Rosalind Eeles, David C Wedge, Peter Van Loo, Gunes Gundem, Ludmil B Alexandrov, Barbara Kremeyer, Adam Butler, Andrew G Lynch, Niedzica Camacho, Charlie E Massie, Jonathan Kay, Hayley J Luxton, Sandra Edwards, Zsofia Kote-Jarai, Nening Dennis, Sue Merson, Daniel Leongamornlert, Jorge Zamora, Cathy Corbishley, Sarah Thomas, Serena Nik-Zainal, Manasa Ramakrishna, Sarah O’Meara, Lucy Matthews, Jeremy Clark, Rachel Hurst, Richard Mithen, Robert G Bristow, Paul C Boutros, Michael Fraser, Susanna Cooke, Keiran Raine, David Jones, Andrew Menzies, Lucy Stebbings, Jon Hinton, Jon Teague, Stuart McLaren, Laura Mudie, Claire Hardy, Elizabeth Anderson, Olivia Joseph, Victoria Goody, Ben Robinson, Mark Maddison, Stephen Gamble, Christopher Greenman, Dan Berney, Steven Hazell, Naomi Livni, the ICGC Prostate Group, Cyril Fisher, Christopher Ogden, Pardeep Kumar, Alan Thompson, Christopher Woodhouse, David Nicol, Erik Mayer, Tim Dudderidge, Nimish C Shah, Vincent Gnanapragasam, Thierry Voet, Peter Campbell, Andrew Futreal, Douglas Easton, Anne Y Warren, Christopher S Foster, Michael R Stratton, Hayley C Whitaker, Ultan McDermott, Daniel S Brewer \& David E Neal Nat. Genet. 47, 367-372 (2015); published online 2 March 2015; corrected after print 5 May 2015

In the version of this article initially published, author Manasa Ramakrishna was omitted from the author list. The error has been corrected in the PDF and HTML versions of this article.

\section{Corrigendum: A common variant mapping to CACNA1A is associated with susceptibility to exfoliation syndrome}

Tin Aung, Mineo Ozaki, Takanori Mizoguchi, R Rand Allingham, Zheng Li, Aravind Haripriya, Satoko Nakano, Steffen Uebe, Jeffrey M Harder, Anita S Y Chan, Mei Chin Lee, Kathryn P Burdon, Yury S Astakhov, Khaled K Abu-Amero, Juan C Zenteno, Yildirim Nilgün, Tomasz Zarnowski, Mohammad Pakravan, Leen Abu Safieh, Liyun Jia, Ya Xing Wang, Susan Williams, Daniela Paoli, Patricio G Schlottmann, Lulin Huang, Kar Seng Sim, Jia Nee Foo, Masakazu Nakano, Yoko Ikeda, Rajesh S Kumar, Morio Ueno, Shin-ichi Manabe, Ken Hayashi, Shigeyasu Kazama, Ryuichi Ideta, Yosai Mori, Kazunori Miyata, Kazuhisa Sugiyama, Tomomi Higashide, Etsuo Chihara, Kenji Inoue, Satoshi Ishiko, Akitoshi Yoshida, Masahide Yanagi, Yoshiaki Kiuchi, Makoto Aihara, Tsutomu Ohashi, Toshiya Sakurai, Takako Sugimoto, Hideki Chuman, Fumihiko Matsuda, Kenji Yamashiro, Norimoto Gotoh, Masahiro Miyake, Sergei Y Astakhov, Essam A Osman, Saleh A Al-Obeidan, OhoudOwaidhah, Leyla Al-Jasim, Sami Al Shahwan, Rhys A Fogarty, Paul Leo, Yaz Yetkin, Çilingir Oğuz, Mozhgan Rezaei Kanavi, Afsaneh Naderi Beni, Shahin Yazdani, Evgeny L Akopov, Kai-Yee Toh, Gareth R Howell, Andrew C Orr, Yufen Goh, Wee Yang Meah, Su Qin Peh, Ewa Kosior-Jarecka, Urszula Lukasik, Mandy Krumbiegel, Eranga N Vithana, Tien Yin Wong, Yutao Liu, Allison E Ashley Koch, Pratap Challa, Robyn M Rautenbach, David A Mackey, Alex W Hewitt, Paul Mitchell, Jie Jin Wang, Ari Ziskind, Trevor Carmichael, RangappaRamakrishnan, Kalpana Narendran, Rangaraj Venkatesh, Saravanan Vijayan, Peiquan Zhao, Xueyi Chen, Dalia Guadarrama-Vallejo, Ching Yu Cheng, Shamira A Perera, Rahat Husain, Su-Ling Ho, Ulrich-Christoph Welge-Luessen, Christian Mardin, Ursula Schloetzer-Schrehardt, Axel M Hillmer, Stefan Herms, Susanne Moebus, Markus M Nöthen, Nicole Weisschuh, Rohit Shetty, Arkasubhra Ghosh, Yik Ying Teo, Matthew A Brown, Ignacio Lischinsky, Blue Mountains Eye Study GWAS Team, Wellcome Trust Case Control Consortium 2, Jonathan G Crowston, Michae Coote, Bowen Zhao, Jinghong Sang, Nihong Zhang, Qisheng You, Vera Vysochinskaya, Panayiota Founti, Anthoula Chatzikyriakidou, Alexandros Lambropoulos, Eleftherios Anastasopoulos, Anne L Coleman, M Roy Wilson, Douglas J Rhee, Jae Hee Kang, Inna May-Bolchakova, Steffen Heegaard, Kazuhiko Mori, Wallace L M Alward, Jost B Jonas, Liang Xu, Jeffrey M Liebmann, Balram Chowbay, Elke Schaeffeler, Matthias Schwab, Fabian Lerner, Ningli Wang, Zhenglin Yang, Paolo Frezzotti, Shigeru Kinoshita, John H Fingert, Masaru Inatani, Kei Tashiro, André Reis, Deepak P Edward, Louis R Pasquale, Toshiaki Kubota, Janey L Wiggs, Francesca Pasutto, Fotis Topouzis, Michael Dubina, Jamie E Craig, Nagahisa Yoshimura, Periasamy Sundaresan, Simon W M John, Robert Ritch, Michael A Hauser \& Chiea-Chuen Khor Nat. Genet. 47, 387-392 (2015); published online 23 February 2015; corrected online 9 March 2015; corrected after print 27 April 2015

In the version of this article initially published, the name of author Afsaneh Naderi Beni was misspelled. The error has been corrected in the HTML and PDF versions of the article. 